\title{
Schnell und prozesssicher durch maschinelle Reinigung
}

\author{
Spezielle Einsätze für Werkzeughalter und Werkzeugaufnahmen, die in Reinigungskörben platziert \\ werden können, ermöglichen eine schnelle und sichere maschinelle Reinigung sowie den Transport \\ der bestückten Werkzeugaufnahmen.
}

Ob Bohren, Fräsen oder Drehen - Verschmutzungen weisen nicht nur die gefertigten Bauteile auf, sondern auch die Werkzeuge und Aufnahmen. Saubere Werkzeugaufnahmen leisten einen wichtigen Beitrag zur Präzision in der Zerspanung. Aber obwohl für die Reinigung der gefertigten Teile meist eine Reinigungsanlage vorhanden ist, werden die Aufnahmen häufig noch manuell gereinigt.

Dabei ist jedoch nicht gewährleistet, dass alle Späne und Reste des Bearbeitungsmediums zuverlässig entfernt werden. Ein weiterer Nachteil ist der hohe Personal- und Zeitaufwand, den jeder manuelle Prozess verursacht. Hinzu kommt, dass die Reinigung mit kostspieliger Druckluft oder Pinsel und leicht flüchtigen Lösemitteln wie Waschbenzin erfolgt.

\section{Spezielle Einsätze für Reinigungskörbe}

Um die vorhandene Anlage auch für die Reinigung von Werkzeughaltern und bestückten Werkzeugaufnahmen nutzen zu können, hat Metallform spezielle Einsätze für Standard-Reinigungskörbe entwickelt. Sie sind für unterschiedliche Aufnahmen wie beispielsweise HSK, Konus, SBA und VDI ausgelegt und können entsprechend dem Typ beziehungsweise der Größe der Werkzeugaufnahme ausgeführt werden. Die Aufnahmen werden dabei nur an unkritischen Bereichen fixiert. Abgestimmt auf die Größe der Arbeitskammer der Reinigungsanlage werden die bestückten Werkzeugaufnahmen senkrecht oder horizontal - beispielsweise bei Tieflochbohrern - darin aufgenommen.

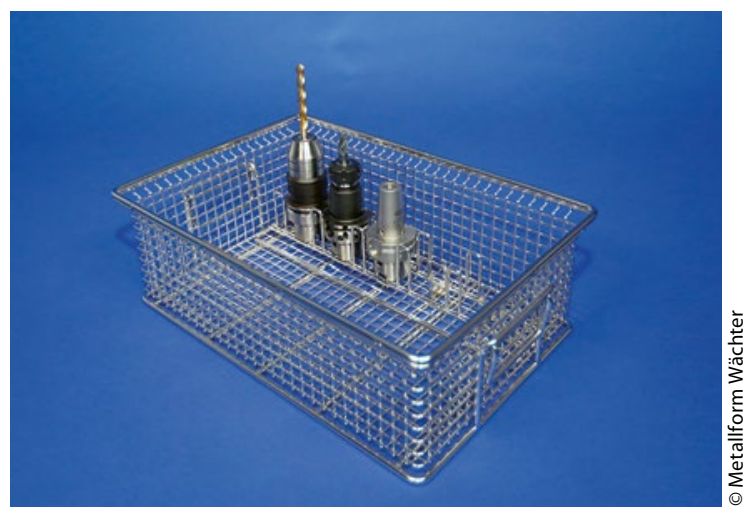

\author{
Für die Reinigung werden \\ die Einsätze in Standard- \\ Reinigungskörben fixiert. \\ Entsprechend den \\ Abmessungen der \\ Arbeitskammer der \\ Reinigungsanlage können \\ mehrere Einsätze in einem \\ Korb platziert werden.
}

Auf diese Weise können die mit Werkzeugen bestückten Aufnahmen direkt an der Maschine oder dem Bearbeitungszentrum in der Einsatzhorde platziert und zur Reinigungsanlage transportiert werden. Für die Reinigung lassen sich mehrere, auch unterschiedliche Einsätze in einem Standard-Reinigungskorb zu einer Charge zusammenfassen. Die Reinigung der Aufnahmen wird somit prozesssicher und schneller.

\section{Hohe Reinigungswirkung und lange Nutzungsdauer}

Die offene Gestaltung der Einsätze stellt eine gute Zugänglichkeit von Medium und Waschmechanik zu den zu reinigenden Teilen sicher. Um die Reinigungswirkung zu verbessern, können die Körbe in der Anlage geschwenkt werden. Nach der Reinigung und Neubestückung lassen sich die Aufnahmen in den Einsätzen wieder sicher und beschädigungsfrei zur Maschine transportieren.
Die Einsätze werden aus rostfreiem Edelstahl mit elektropolierter Oberfläche gefertigt und können daher mit allen Reinigungsmedien eingesetzt werden. Außerdem ermöglicht das Material eine lange Nutzungsdauer. Die Rundstäbe der äußeren Struktur sind stumpf verschweißt, wodurch verletzungsgefährliche Ecken vermieden werden. //

\section{Kontakt}

Metallform Wächter GmbH

Bretten

Jörg Schleeh, Tel. 07252942636

joerg.schleeh@metallform.de

www.metallform.de 